\title{
Minireview
}

\section{Establishing a Link between Oncogenes and Tumor Angiogenesis}

\author{
Robert S. Kerbel, Alicia Viloria-Petit, Futoshi Okada, and \\ Janusz Rak \\ Biological Sciences Program, Division of Cancer Biology Research, \\ Sunnybrook Health Science Centre, Toronto, Ontario, Canada \\ Department of Medical Biophysics, University of Toronto, Toronto, \\ Ontario, Canada
}

The progressive growth and expansion of solid tumors beyond microscopic sizes of about $1-2$ $\mathrm{mm}$ in diameter requires the formation of new blood vessels-a process known as tumor angiogenesis. Two major types of event are thought to be involved in the ability of tumors to switch on the angiogenic phenotype: a gain-of-function event in which various stimulators of angiogenesis are induced, or up-regulated, in tumor cells, and a loss-of-function event involving downregulation of one or more endogenous inhibitors of angiogenesis (1). Activation of oncogenes and inactivation of tumor suppressor genes are generally associated with gain- and loss-of-function events, respectively (2). Hence, these types of genetic alterations could be one of the main ways in which the process of tumor angiogenesis is switched on and sustained. This has potentially important therapeutic implications for the use of drugs designed to target and inhibit the overexpressed proto-oncogenes or mutant oncogenes or their encoded oncoproteins. In short, such drugs may function in vivo, at least in part, as inhibitors of tumor angiogenesis-an anti-tumor effect that would be missed in tissue culture assays used for drug development and activity.

Address correspondence and reprint requests to: Dr. Robert S. Kerbel, Biological Sciences Program, Division of Cancer Biology Research, Sunnybrook Health Science Centre, Research Building, S-218, 2075 Bayview Avenue, Toronto, Ontario M4N 3M5 Canada. Phone: (416) 480-5711; Fax: (416) 480-5703; E-mail: kerbel@srcl.sunnybrook.utoronto

\section{Positive and Negative Regulators of Tumor Angiogenesis}

Folkman first put forward the hypothesis that the growth of solid tumors is "angiogenesis dependent" $(3,4)$, which is the basis for anti-cancer treatment strategies aiming to develop drugs that selectively target newly formed, immature blood vessels in tumors while leaving normal mature vessels elsewhere in the body unharmed $(3,4)$. This hypothesis has stood the test of time, at least in preclinical models of tumor growth and therapy $(5,6)$. There is now great interest in academic research laboratories and in the biotech/pharmaceutical industry in the development of drugs that inhibit or enhance angiogenesis. There are a number of reasons for this interest. (1) The possibility exists that resistance to certain angiogenesis inhibitors may not develop in tumor cells exposed to such drugs, even over prolonged periods of time (7-9). (2) A growing number of molecular targets on "activated" endothelial cells have been identified that are associated with newly formed blood vessel capillaries, such as acutely up-regulated receptor tyrosine kinases (10-12), integrins (13), and adhesion molecules $(14,15)$. (3) A number of potentially powerful endogenous protein inhibitors of angiogenesis that can cause tumor regressions (16) and are usually internal fragments of higher molecular weight proteins have been discovered (16). (4) There is a growing sense of urgency about the need to devise new and innovative anti-cancer strategies or drugs to replace or supplement the 
ones that have been used with such limited success for the past 50 years.

When Folkman first put forward his seminal hypothesis more than 25 years ago, he initially envisioned a scenario in which developing tumor masses remained microscopic and dormant as long as they were incompetent to induce angiogenesis $(3,4)$. He suggested that termination of this dormant phenotype was brought about by the induction and release of soluble and diffusable growth factor, which he called tumor angiogenic factor (TAF) $(3,4)$. Release of this hypothetical molecule into the extracellular environment would set in motion the various chains of events associated with the formation of new blood vessel capillaries sprouting from the mature and pre-existing host vasculature located in the vicinity of a TAF-producing tumor mass. These events include localized proteolysis of the basement membranes surrounding mature blood vessels, migration of endothelial cells through the newly created breach, endothelial cell proliferation, the formation of rudimentary tubes or vascular sprouts, and the joining of such newly formed vessels to form a vascular network $(1,6)$. It took another 15 years before a molecule that could be equated with TAF was identified. It turned out to be basic fibroblast growth factor (bFGF), also known as FGF-2 $(17,18)$. Since then, at least a dozen more growth factors have been identified as having potential tumor angiogenesis promoting activity (6). These include growth factors also known to have mitogenic autocrine activity for tumor cells, such as transforming growth factor alpha (TGF- $\alpha$ ), and a number of tumor- or stromal cell-derived paracrine growth factors (for endothelial cells), including vascular endothelial cell growth factor VEGF, which is also known as vascular permeability factor (VPF); scatter factor (SF), also known as hepatocyte growth factor (HGF); transforming growth factor beta (TGF- $\beta$ ); angiopoietin 1 and 2; platelet-derived growth factor (PDGF); and interleukin-8 (IL-8), among others (6). In addition to these growth factors, whose expression can be induced or up-regulated in tumor cells, there is a family of angiogenesis inhibitory molecules, some of which are now known to be strongly down-regulated in tumor cells. Thrombospondin- 1 is perhaps the best example of such an inhibitor (6). Bouck and colleagues first reported evidence that the wild-type $p 53$ gene is a positive regulator of thrombospondin expression; inactivation of $p 53$ by mu- tational or deletion events can result in loss of thrombospondin expression (19). Indeed, this led Bouck to propose that a major consequence of inactivation of wild-type suppressor genes would be the facilitation of tumor angiogenesis, primarily by eliminating or reducing the expression of endogenous inhibitors of angiogenesis (20). This would contribute (indirectly) to the tumor-promoting function of such genetic alterations, in addition to their direct effects on enhancing unrestricted cell proliferation and cell survival $(6,19)$.

\section{The Connection of Oncogenes to Tumor Angiogenesis}

A survey of some of the growth factors up-regulated in tumor cells immediately suggests a possible role for oncogenes in tumor angiogenesis. For example, both TFG- $\alpha$ and TGF- $\beta$, which are proangiogenic molecules in vivo (6), are known to be induced or up-regulated in mutant ras transformed cells $(21,22)$. The same is true for bFGF (23). Two other considerations led us to speculate in 1995 that a major, unappreciated function of oncogenes is to contribute to the angiogenic phenotype in tumors. First, it is known that the growth fraction of many solid tumors is actually quite low $(24,25)$, in some cases surprisingly so, as in rapidly expanding metastatic prostate cancer deposits growing in the bone (26). The low fractions of solid tumors is thought to be a major source of the intrinsic resistance characteristics of such tumors to cytotoxic chemotherapeutic drugs, which generally target rapidly dividing cells (27). This presents an interesting paradox, given the emphasis that has been placed on aberrant cell cycle regulation ("unrestricted cell proliferation") as the predominant functional effect of most oncogenes on the transformed phenotype. For example, because metastatic prostatic cancers have tumor cell growth fractions in the range of only $2 \%(26)$, it is difficult to accept the idea that oncogenes that contribute to prostate cancer do so primarily, if at all, through their effects on directly promoting aberrant cell proliferation. In this regard, it is worth pointing out that when tumor cells are grown in monolayer cell culture, it is not uncommon to encounter growth fractions in the range of $75 \%$, which is far greater than that observed in vivo (27). However, growth in vitro as threedimensional multicellular spheroids can lead to a 
very significant reduction in the growth fraction (27), despite the presence of numerous inactivated tumor suppressor genes and mutant oncogenes in the cultured tumor cells. Perhaps the predominant use of monolayer cell culture systems to study cancer biology in vitro has resulted in a somewhat distorted view of the relative importance and contribution of uncontrolled cellcycle proliferation to the overall growth and expansion of solid tumors in vivo (27). If so, the following question emerges: how do oncogenes contribute to the ability of tumors to grow indefinitely in addition to, or instead of, promoting aberrant cell proliferation? Could an indirect mechanism of growth promotion be involvednamely, induction of or contribution to angiogenesis?

A second paradoxical observation that caught our attention concerns the therapeutic effects of a new class of anti-cancer drugs generally known as signal transduction inhibitors. The best known examples of such drugs include small-molecular-weight Ras farnesyltransferase inhibitors (FTIs) $(28,29)$ and monoclonal neutralizing antibodies to overexpressed cell-surface receptor tyrosine kinases, such as the EGF receptor or erbB2/HER-2/neu $(30,31)$. In general, such drugs are considered to be cytostatic rather than cytotoxic agents because they inhibit growth of target tumor cells in (monolayer) cell culture in the absence of any significant killing $(28,29,32)$. Moreover, the cytostatic effects are often modest-in the range of $30-60 \%$ inhibition at the highest drug concentrations $(28,29,32)$. Consequently, a reasonable prediction would be that such drugs possess only modest anti-tumor effects in vivo when tested on established tumors, i.e., overt regressions of tumor mass would not be a feature of treatment with such drugs. Rather, tumors would be kept from expanding by an induced state of dormant growth. Surprisingly, this is not necessarily the case (32-34). For example, regression of established experimental tumors in mice has been observed, in some cases, by using Ras FTIs on certain transgenic "oncomouse" strains (33). Moreover, the extent and rapidity of tumor regressions can match or even exceed maximum tolerated doses of conventional cytotoxic anticancer drugs such as adriamycin (33).

How can such an unexpected discrepancy be accounted for? One possible explanation is that the agents may be found to be cytotoxic when tested against tumor cells grown in a solid tumor (multicellular) context, rather than in monolayer cell cultures, which is the usual way tumor cells are grown for drug-testing studies in cell culture. Indeed, there is evidence that ras oncogenes can function as potent survival factors by suppressing the massive levels of apoptosis of epithelial cells observed when such cells are grown nonphysiologically as multicellular spheroids (35). This probably explains why Ras FTIs can induce apoptosis in ras-transformed cells grown anchorage independently, but not in monolayer cell culture (36), and as it now turns out, in cells grown in vivo, as established solid tumors (37).

However, an alternative or additional mechanism of cell killing by such signal transduction inhibitors could involve inhibition of tumor angiogenesis, as we first suggested in 1995 (38) and as schematically outlined in Figure 1. If it is supposed that activation of an oncogene such as ras leads to a marked induction in tumors cells of a paracrine-acting angiogenesis growth factor such as VEGF/VPF, treatment of such cells with a drug such as a Ras FTI could result in down-regulation or suppression of that tumor cell-derived angiogenesis factor. This in turn could endow the drug with potential anti-angiogenic properties, which could then lead to an increase in tumor cell apoptosis, because blocked angiogenesis is often associated with an increase in the levels of apoptotic cells detected in tumors $(39,40)$. Moreover, even modest (2- to 3 -fold) reductions in VEGF/VPF expression can be associated with profound degrees of tumor growth inhibition (41) as well as endothelial cell apoptosis $(42,43)$, leading to blood vessel destruction $(42,44)$. The latter could lead to the former (42).

In an effort to test these hypotheses, we initiated a series of experiments designed to determine first, whether mutant ras oncogenes, and subsequently, other oncogenes, could act as inducers of VEGF/VPF gene and protein expression. Assuming such an association could be uncovered, we could then test the effects of various signal transduction anti-tumor inhibitory drugs for their effects on VEGF/VPF expression both in vitro and in vivo against appropriate target tumor cells. Our first experiments utilized IEC-18 cells, which is a spontaneously immortalized cell line of rat intestinal epithelial origin totally incapable of forming tumors in nude mice (38). A number of clonal ras-transformed sublines were obtained by transfection of IEC-18 cells with a mutant human $\mathrm{H}$-ras oncogene (38), all of which 


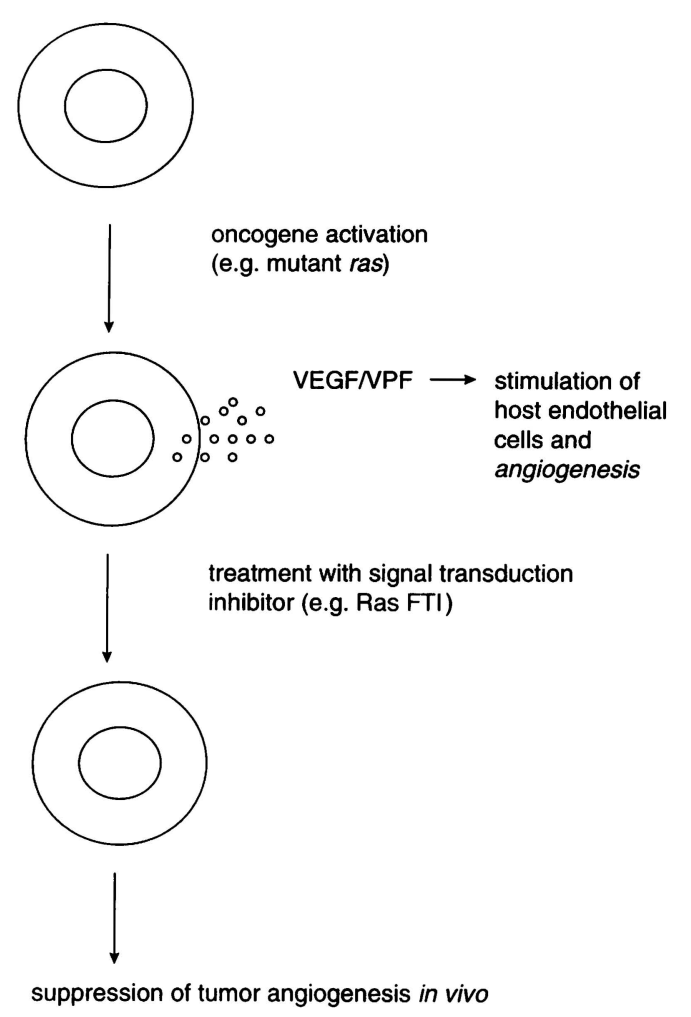

Fig. 1. Outline of how oncogenes and signal transduction inhibitor drugs may contribute to induction and inhibition of tumor angiogenesis, respectively. Oncogene (e.g., ras) activation can lead to induction of VEGF/VPF, a potent mediator of angiogenesis. VEGF/VPF cannot function as an autocrine growth factor for tumor cells as tumor cells generally lack receptors for VEGF/VPF. In contrast, activated endothelial cells can express high levels of VEGF/VPF, perhaps because of the inductive effect of VEGF/VPF itself. Treatment of the VEGF/ VPF-positive tumor cells with a signal transduction inhibitor e.g., a Ras farnesyltransferase inhibitor (FTI), can lead to, among many other changes, a reduction in VEGF/VPF expression. This could in turn lead to a suppressed in vivo angiogenic response. Oncogene activation could also lead to expression of growth factors having both autocrine and paracrine/ angiogenesis-promoting functions (such as TGF- $\alpha$ ) and/or down-regulation of angiogenesis inhibitory molecules, such as thrombospondin (see text).

were found to be highly tumorigenic in nude mice, e.g., the IEC-ras3, IEC-ras4, and IEC-ras7 cell lines (38). As shown in Figure 2, the parental IEC-18 cell line was essentially negative for VEGF/VPF gene expression, as assessed by Northern blotting experiments (38). In marked and obvious contrast, the ras-transformed sublines were all strongly VEGF/VPF positive. This included a clone in which mutant $\mathrm{H}$-ras was put under the control of heavy metal inducible pro-
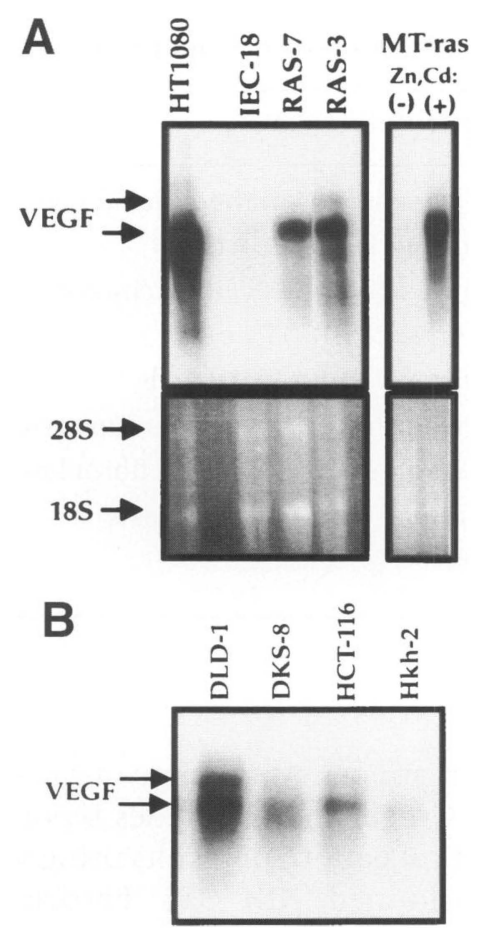

Fig. 2. Mutant ras oncogene regulates expression of VEGF mRNA. (A) Northern blot of VEGF mRNA is shown of human HT1080 human fibrosarcoma cells (as a positive control), nontumorigenic rat intestinal epithelial cell line-18 (IEC-18 cells), and two clones of IEC-18, Ras-7 and Ras-3, which were obtained by transfection of IEC-18 cells with a mutant human H-ras oncogene. These latter two lines are tumorigenic, and unlike IEC-18 cells, they express abundant VEGF transcripts. MT-ras is a clone of IEC-18 which expresses its transfected mutant H-ras oncogene only when exposed to heavy metals such as zinc and cadmium, as the gene is under the control of a metallothionein promotor. VEGF mRNA are not expressed in this clone unless they are exposed to these metals. (B) Northern blot for VEGF mRNA in human DLD-1 and HCT-116 human colorectal carcinoma cells, each of which contains a single mutant K-ras allele. DKS- 8 and Hkh-2 are sublines obtained from DLD-1 and HCT-116, respectively, in which the mutant $\mathrm{K}$-ras allele has been disrupted by gene targeting (55). The DLD-1 and HCT-116 cell lines are tumorigenic in nude mice, whereas DKS- 8 and Hkh-2 are not. The suppression of VEGF mRNA is matched by a down-regulation in VEGF protein released by the cells into the conditioned medium. The results are taken from Rak et al. (38).

moter-so long as the cells were exposed to zinc and cadmium (38), as shown in Figure 2. Similarly, IEC-18 cells transfected with a tetracyclinregulated ras gene expression construct were VEGF/VPF mRNA positive in the absence of tetracyclin and negative in its presence (unpub- 
TABLE 1. Mutant ras oncogene induction or up-regulation of VEGF/VPF

\begin{tabular}{ll}
\hline Cells/System & \multicolumn{1}{c}{ Reference } \\
\hline H-ras-transformed intestinal epithelial cells & Rak et al., 1995 (38) \\
v-ras-transformed NIH-3T3 cells & Grugel et al., 1995 (45) \\
H-ras in mouse squamous cell carcinomas & Larcher et al., 1996 (48) \\
H-ras transformed NIH-3T3 cells & Mazure et al., 1996 (47) \\
H-ras-transformed endothelial cells & Arbiser et al., 1997 (52) \\
H-ras in hamster buccal pouch keratinocytes & Lingen et al., 1997 (51) \\
H-ras in Li Fraumeni p53-human fibroblasts & Volpert et al., 1997 (49) \\
v-H-ras in human IMR-90 fibroblasts & Enholm et al., 1997 (53) \\
v-H-ras in NIH-3T3 cells & White et al., 1997 (54)
\end{tabular}

lished observations). At the time our results were published, Grugel and colleagues reported a virtually identical pattern of results using v-ras (and v-raf) transformed NIH 3T3 fibroblasts (45). Since then, a number of confirmatory reports have appeared showing an association between ras expression, which is often, but not always, mediated by mutant ras oncogene transfection, and induction or up-regulation of VEGF/VPF gene expression in mouse, rat, hamster, and human cells of variable origin $(46-54)$. This is summarized in Table 1 . In virtually all of these experiments, the increase in mRNA expression was matched by a commensurate increase in protein expression.

An alternative and complimentary method to demonstrate a cause-and-effect relationship between oncogenic ras mutations is to examine VEGF/VPF expression in VEGF/VPF-positive human colorectal carcinomas that carry a single mutant K-ras allele, and sublines of such tumors in which the dominantly acting mutant K-ras allele is disrupted by gene-targeting methods (38). Such sublines have been obtained by Shirasawa et al. from the highly tumorigenic HCT-116 and DLD-1 human colorectal carcinomas (55). It is remarkable that the sublines containing a disrupted K-ras allele were found to be nontumorigenic in nude mice $(38,44,55)$ despite the retention of the numerous other genetic alterations normally associated with, and presumed to be causative of, advanced colon cancer. Could this profound loss in tumorigenicity be related to a marked suppression of VEGF/VPF expression in the mutant K-ras knockout sublines? We have speculated that the answer to this question is an affirmative one, based on several findings (44).
First, the knockout sublines were found to express an approximately 4-fold reduction in VEGF/VPF mRNA (see Fig. 2) and protein levels (44). Second, a similar reduction in VEGF/VPF expression mediated by transfection of HCT-116 or DLD-1 cells with a VEGF ${ }_{121}$ antisense cDNA expression construct resulted in the derivation of several clones from each parent line which were suppressed 2- to 4-fold for VEGF/VPF protein expression (44). All of these clones were profoundly suppressed in their tumor-forming ability in nude mice, grow normally in cell culture. Third, when VEGF/VPF-deficient mutant K-ras knockout sublines were used as recipients for a $\mathrm{VEGF} / \mathrm{VPF}_{121}$ "sense" transfection procedure, a number of the VEGF/VPF-expressing variants showed a weak but detectable increase in tumorforming ability in nude mice but no growth advantage in tissue culture (44). Taken together, these results suggest that ras oncogene-induced VEGF/VPF expression is necessary, but clearly not sufficient, for aggressive tumorigenic growth in vivo. This conclusion would appear to make intuitive sense because knocking out the mutant ras allele would lead to suppression of numerous and different pro-cell transformation events involving, for example, growth, invasion, and survival, in addition to angiogenesis. Restoring some degree of VEGF/VPF expression would not affect these other vital, transformed cell-associated phenotypes. Indeed, even the degree of angiogenesis competence that is restored by a VEGF $_{121}$ transfection method might be an underestimate, since other VEGF/VPF isoforms (e.g., $V_{E G F_{165}}$ ) and additional pro-angiogenic growth factors (e.g., bFGF, IL-8, etc.) suppressed in the knockout sublines would not have their 
levels restored by transfection of a sense VEGF/ $\mathrm{VPF}_{121}$ cDNA expression construct (44).

More recently, we have found that other genetic alterations that effectively function as dominant oncogenes, e.g., overexpression of the EGF and erbB2/neu/Her2 receptor tyrosine kinases, are also associated with induction or upregulation of VEGF/VPF mRNA and/or protein expression (56). This is consistent with the previous results of some other studies showing, for example, that TGF- $\alpha$ or IGF-1 can induce VEGF/ VPF expression in vitro $(57,58)$. It is also now known that other classes of oncogenes, e.g., genes that encode transcription factors such as c-fos (46) or protein translational initiation factors such as ElF-4e (59), can function as potent inducers of VEGF/VPF expression both in vitro and in vivo (46). Thus a generic function of many oncogenes may be to promote tumor growth and survival indirectly through an angiogenesis-dependent mechanism, as well as directly through their effects on enhancing intrinsic tumor cell proliferation and survival. This suggests that various signal transduction inhibitors being developed as anti-cancer drugs may function in vivo as de facto anti-angiogenic agents.

\section{Signal Transduction Inhibitors as Anti-Tumor Agents: Do They Inhibit Tumor Angiogenesis In Vivo?}

This is a difficult question to answer in a definitive manner, but the evidence obtained thus far would appear to indicate that one possible effect of administering a variety of signal transduction inhibitors to tumor-bearing mice would be to suppress VEGF/VPF expression and in all probability, a number of other pro-angiogenic growth factors as well, thereby endowing such drugs with the potential to block or suppress tumor growth made of tumor cells by inhibiting the angiogenesis competence of the treated tumors.

Our first attempt at analyzing this question involved an examination of the effects of Ras FT1 called L-739, 749 (38) on VEGF/VPF expression using VEGF/VPF cultured ras-transformed IEC-1 8 cells as a target population in vitro (38). The results showed that one effect of drug treatment of such cells in vitro was down-regulation of VEGF/VPF expression (38). We have not yet determined whether a similar effect of the drug would be observed in vivo in drug-treated tumor-bearing mice.
However, such an in vivo effect has been observed with a different class of anti-tumor signal transduction inhibitory agent, namely, monoclonal neutralizing antibodies to the human EGF receptor (56). We had found that human A431 squamous carcinoma cells, which overexpress the EGF receptor, displayed reduced (up to 2-fold) levels of VEGF/VPF mRNA and protein in vitro after treatment in culture with varying concentrations of the EGF receptor neutralizing antibody, which is known as C225 (32). We therefore attempted a similar experiment with A431 squamous carcinoma cells grown as subcutaneous xenografts in nude mice (56). For these experiments, nude mice were injected with A431 cells and the tumors allowed to grow for approximately 3-4 weeks. One group of mice was injected four times with C225 intraperitoneally (one injection every 2 days) and the tumors were removed shortly after the last injection. They were assessed for their relative expression of VEGF/VPF protein by immunostaining, in comparison to the control mice. The results showed a rather striking down-regulation of VEGF/VPF expression in the A431 tumors removed from mice that had been given the $\mathrm{C} 225$ antibody (56). The level of reduction could not be quantitated with accuracy but appear to exceed 3-fold (56). As discussed above, this level of suppression, when induced by an antisense method, can have profound suppressive consequences on tumor growth in vivo in the absence of any anti-tumor effect in cell culture $(41,44)$.

Thus, it would seem reasonable to postulate that long-term therapy of tumors with an agent such as C225 could lead to an indirect mechanism of suppression of tumor growth as a result of blocked angiogenesis. This may be true of many drugs designed to inhibit the expression of different classes of mutant or overexpressed oncoproteins. If so, it could partially explain why such drugs appear to be more potent in vivo than one would anticipate from their behavior as antitumor drugs on cells grown in monolayer cell culture where generally only modest and noncytotoxic effects are observed. In this respect, it would be of interest to determine whether the putative anti-angiogenic effects of such agents can be separated from their direct anti-proliferative effects on tumor cells. Are the drug concentrations and scheduling that are optimal for inhibiting (directly) tumor cell growth (or survival) the same as those for inhibiting angiogenesis? 


\section{Oncogenes (and Tumor Suppressor Genes) and Angiogenesis Inhibitors}

The emphasis in this discussion has been on the idea that oncogenes can contribute to tumor angiogenesis primarily by virtue of their stimulatory effects on the expression of pro-angiogenic growth factors such as VEGF/VPF. However, as discussed earlier, the angiogenic switch is also affected by the loss of angiogenesis inhibitors such as thrombospondin- 1 as a result of inactivation of the $p 53$ gene (19). It is conceivable that oncogenes could contribute to the angiogenic switch by causing a similar down-regulation of various angiogenesis inhibitors. Indeed, several groups have reported that the levels of thrombospondin in ras oncogene-transformed fibroblasts can be strongly suppressed $(60,61)$. Likewise, inactivation of the von Hippel Lindau suppressor gene can lead to a marked induction or up-regulation of VEGF/VPF gene and protein expression $(62,63)$. Hence it is becoming clear that the ways in which mutant or deleted tumor suppressor genes and oncogenes can influence angiogenesis is not only by loss of angiogenesis inhibitors and induction of angiogenesis stimulators, respectively.

\section{Interaction of Oncogenes with Physiologic Regulators of Tumor Angiogenesis}

One important and potent mediator of VEGF/ VPF expression both in vitro and in vivo is reduced oxygen concentrations, i.e., hypoxia $(64,65)$. This effect of hypoxia is mediated both by a transcriptional effect and an increase in mRNA stability $(66)$; the latter seems to be the more important $(66,67)$. This has led to the view that physiologic stresses such as hypoxia in solid tumors may be the major inducing influence of angiogenesis rather than genetic changes per se (68). It is becoming increasingly evident, however, that a combination of genetic and epigentic (i.e., hypoxia) changes can function together in a synergistic manner to boost VEGF/VPF expression in tumor cells $(47,52,56)$. This effect, as observed in mutant neu oncogene-transformed NIH 3 T3 fibroblasts, can be quite dramatic (56). The signalling pathways that are involved in this interaction are now being analyzed $(47,50)$. For example, activation of P1 3 kinase, but not MAP kinases, has been implicated in combined onco- gene-hypoxia induction of VEGF/VPF in rastransformed fibroblasts (50).

\section{Summary}

We have tried to stress that mutant oncogenes or overexpressed, nonmutateci proto-oncogenes, in addition to their direct affect on promoting aberrant tumor cell proliferation (and survival), may possess a crucial indirect means of stimulating tumor cell growth through regulation of angiogenesis. This effect would never be observed in tissue culture studies of oncogene function using pure cultures of tumor cells, which probably helps explain why the pro-angiogenic function of oncogenes has not been appreciated until only relatively recently. Indeed, the very first indication of a possible contributory role of oncogenes, such as ras and myc, to tumor angiogenesis was first reported by Thompson et al. in 1989, who used reconstituted organ cultures of the mouse prostate gland for their studies (69). This potentially important contribution of oncogenes to tumor growth and development may prove to have an impact on how various signal transduction inhibitors that are now in early phase clinical trials, e.g., monoclonal neutralizing antibodies to the human EGF receptor (70), function in vivo as anti-tumor agents.

\section{Acknowledgments}

We are grateful to Lynda Woodcock and Cassandra Cheng for their excellent secretarial assistance. R. S. K.'s angiogenesis-related research is supported by grants from the Medical Research Council of Canada and the National Institutes of Health, U.S.A. (CA41223). R. S. K. is a Terry Fox Scientist of the National Cancer Institute of Canada.

\section{Note Added in Proof}

Another interesting example of the impact of oncogenes on tumor angiogenesis was reported by Bais et al. (71) who found that the G-proteincoupled receptor of Kaposi's sarcoma-associated herpesvirus is both a viral oncogene and angiogenesis activator. 


\section{References}

1. Hanahan D, Folkman J. (1996) Patterns and emerging mechanisms of the angiogenic switch during tumorigenesis. Cell 86: 353-364.

2. Fearon ER, Vogelstein B. (1990) A genetic model for colorectal tumorigenesis. Cell 61: 759-767.

3. Folkman J. (1971) Tumor angiogenesis: therapeutic implications. New Engl. J. Med. 285: 1 182-1 186.

4. Folkman J. (1972) Anti-angiogenesis: New concept for therapy of solid tumors. Ann. Surg. 175: 409-416.

5. Folkman J. (1990) What is the evidence that tumors are angiogenesis-dependent? J. Natl. Cancer Inst. 82: 4-6.

6. Bouck N, Stellmach V, Hsu SC. (1996) How tumors become angiogenic. Adv. Cancer Res. 69: 135174.

7. Kerbel RS. (1991) Inhibition of tumor angiogenesis as a strategy to circumvent acquired resistance to anti-cancer therapeutic agents. BioEssays 13: 31-36.

8. Boehm T, Folkman J, Browder T, et al. (1997) Antiangiogenic therapy of experimental cancer does not induce acquired drug resistance. Nature 390: 404-407.

9. Kerbel RS. (1997) A cancer therapy resistant to resistance. Nature 390: 335-336.

10. Folkman J, D'Amore PA. (1996) Blood vessel formation: What is its molecular basis? Cell 87: 11531155 .

11. Terman BI, Dougher-Vermazen M. (1996) Biological properties of VEGF/VPF receptors. Cancer Metastasis Rev. 15: 159-163.

12. Thomas KA. (1996) Vascular endothelial growth factor, a potent and selective angiogenic agent. J. Biol. Chem. 271: 603-606.

13. Stromblad S, Cheresh DA. (1998) Cell adhesion and angiogenesis. Trends Cell Biol. 6: 462-468.

14. Brooks PC. (1996) Cell adhesion molecules in angiogenesis. Cancer Metastasis Rev. 15: 187-194.

15. Bischoff J. (1995) Approaches to studying cell adhesion molecules in angiogenesis. Trends Cell Biol. 5: 69-74.

16. Folkman J. (1995) Angiogenesis inhibitors generated by tumors. Mol. Med. 1: 120-122.

17. Baird A, Klagsbrun M. (1991) The fibroblast growth factor family. Cancer Cells 3: 239-243.

18. Rak J, Kerbel RS. (1997) bFGF and tumor angiogenesis-back in the limelight? Nat. Med. 3: 10831084.

19. Dameron KM, Volpert OV, Tainsky MA, et al. (1994) Control of angiogenesis in fibroblasts by p53 regulation of thrombospondin-1. Science 265: 1582-1584.

20. Bouck N. (1990) Tumor angiogenesis: The role of oncogenes and tumor suppressor genes. Cancer Cells 2: 179-185.

21. Cleveland DW, Lopata MA, MacDonald RJ, et al. (1980) Number and evolutionary conservation of alpha- and beta-tubulin and cytoplasmic betaand gamma-actin genes using specific cloned cDNA probes. Cell 20: 95-105.

22. Roberts AB, Sporn MB, Assoian RK, et al. (1986) Transforming growth factor type beta: Rapid induction of fibrosis and angiogenesis in vivo and stimulation of collagen formation in vitro. Proc. Natl. Acad. Sci. U.S.A. 83: 4167-4171.

23. Iberg N, Rogelj S, Fanning P, et al. (1989) Purification of 18- and 22-kDa forms of basic fibroblast growth factor from rat cells transformed by the ras oncogene. J. Biol. Chem. 264: 19951-19955.

24. Tannock I. (1978) Cell kinetics and chemotherapy: A critical review. Cancer Treat. Reports 62: 1117-1133.

25. Olive PL, Durand RE. (1994) Drug and radiation resistance in spheroids: Cell contact and kinetics. Cancer Metastasis Rev. 13: 121-138.

26. Berges RR, Vukanovic J, Epstein J, et al. (1995) Implication of cell kinetic changes during the progression of human prostatic carcinoma. Clin. Cancer Res. 1: 473-480.

27. St.Croix B, Florenes VA, Rak JW, et al. (1996) Impact of the cyclin dependent kinase inhibitor $\mathrm{p} 27^{\text {Kip } 1}$ on adhesion-dependent resistance of tumor cells to anticancer agents. Nat. Med. 2: 12041210.

28. Kohl NE, Mosser SD, deSolms SJ, et al. (1993) Selective inhibition of ras-dependent transformation by a farnesyltransferase inhibitor. Science 260: 1934-1942.

29. James GL, Golstein JL, Brown MS, et al. (1993) Benzodiazepine peptidomimetics: Potent inhibitors of ras farnesylation in animal cells. Science 260: 1937-1942.

30. Mendelsohn J, Fan Z. (1997) Epidermal growth factor receptor family and chemosensitization. J. Natl. Cancer Inst. 89: 341-343.

31. Fendly BM, Winget M, Hudziak RM, et al. (1990) Characterization of murine monoclonal antibodies reactive to either the human epidermal growth factor receptor or HER2/neu gene product. Cancer Res. 50: 1550-1558.

32. Goldstein NI, Prewett M, Zuklys K, et al. (1995) Biological efficacy of a chimeric antibody to the epidermal growth factor receptor in a human tumor xenograft model. Clin. Cancer Res. 1: 13111318.

33. Kohl NE, Omer CA, Conner MW, et al. (1995) Inhibition of farnesyltransferase induces regression of mammary and salivary carcinomas in ras transgenic mice. Nat. Med. 1: 792-797.

34. Ohnishi $Y$, Nakamura $H$, Yoshimura $M$, et al. (1995) Prolonged survival of mice with human gastric cancer treated with an anti-c-erbB-2 monoclonal antibody. Br. J. Cancer 71: 969-973.

35. Rak J, Mitsuhashi Y, Erdos V, et al. (1995) Massive programmed cell death in intestinal epithelial cells induced by three-dimensional growth condi- 
tions: Suppression by expression of a mutant $\mathrm{c}-\mathrm{H}-$ ras oncogene. J. Cell Biol. 131: 1587-1598.

36. Lebowitz P, Sakamuro D, Prendergast GC. (1997) Farnesyl transferase inhibitors induce apoptosis of Ras-transformed cells denied substratum attachment. Cancer Res. 57: 708-713.

37. Barrington RE, Subler MA, Rands E, et al. (1998) A farnesyltransferase inhibitor induces tumor regression in transgenic mice harboring multiple oncogenic mutations by mediating alterations in both cell cycle control and apoptosis. Mol. Cell. Biol. 18: 85-92.

38. Rak J, Mitsuhashi Y, Bayko L, et al. (1995) Mutant ras oncogenes upregulate VEGF/VPF expression: implications for induction and inhibition of tumor angiogenesis. Cancer Res. 55: 4575-4580.

39. Folkman J. (1995) Clinical applications of research on angiogenesis. New Engl. J. Med. 333: 1757-1763.

40. O'Reilly MS, Holmgren L, Chen C, et al. (1996) Angiostatin induces canal sustains dormancy of human tumors in mice. Nat. Med. 2: 689-692.

41. Cheng SY, Huang HJ, Nagane M, et al. (1996) Suppression of glioblastoma angiogenicity and tumorigenicity by inhibition of endogenous expression of vascular endothelial growth factor. Proc. Natl. Acad. Sci. U.S.A. 93: 8502-8507.

42. Benjamin LE, Keshet E. (1997) Conditional switching of vascular endothelial growth factor (VEGF) expression in tumors: induction of endothelial cell shedding and regression of hemangioblastoma-like vessels by VEGF withdrawal. Proc. Natl. Acad. Sci. U.S.A. 94: 8761-8766.

43. Alon T, Hemo I, Itin A, et al. (1995) Vascular endothelial growth factor acts as a survival factor for newly formed retinal vessels and has implications for retinopathy of prematurity. Nat. Med. 1: 1024-1028.

44. Okada F, Rak J, St. Croix B, et al. (1998) Impact of oncogenes on tumor angiogenesis: mutant $\mathrm{K}$-ras up-regulation of VEGF/VPF is necessary but not sufficient for tumorigenicity of human colorectal carcinoma cells. Proc. Natl. Acad. Sci. U.S.A. 35: 3609-3614.

45. Grugel S, Finkenzeller G, Weindel K, et al. (1995) Both v-Ha-ras and v-raf stimulate expression of the vascular endothelial growth factor in NIH 3T3 cells. J. Biol. Chem. 270: 25915-25919.

46. Saez E, Rutberg SE, Mueller E, et al. (1995) c-fos is required for malignant progression for skin tumors. Cell 82: 721-732.

47. Mazure NM, Chen EY, Yeh P, et al. (1996) Oncogenic transformation and hypoxia synergistically act to modulate vascular endothelial growth factor expression. Cancer Res. 56: 3436-3440.

48. Larcher F, Robles AI, Duran H, et al. (1996) Upregulation of vascular endothelial growth factor/ vascular permeability factor in mouse skin carcinogenesis correlates with malignant progression state and activated $\mathrm{H}$-ras expression levels. Cancer Res. 56: 5391-5396.

49. Volpert OV, Dameron KM, Bouck N. (1997) Sequential development of an angiogenic phenotype by human fibroblasts progressing to tumorigenicity. Oncogene 14: 1495-1502.

50. Mazure NM, Chen EY, Laderoute KR, et al. (1997) Induction of vascular endothelial growth factor by hypoxia is modulated by a phosphatidylinositol 3-kinase/Akt signaling pathway in Ha-ras-transformed cells through a hypoxia inducible factor-1 transcriptional element. Blood 90: 3322-3331.

51. Lingen MW, DiPietro LA, Solt DB, et al. (1997) The angiogenic switch in hamster buccal pouch keratinocytes is dependent on TGFbeta- 1 and is unaffected by ras activation. Carcinogenesis 18: 329-338.

52. Arbiser JL, Moses MA, Fernandez CA, et al. (1997) Oncogenic H-ras stimulates tumor angiogenesis by two distinct pathways. Proc. Natl. Acad. Sci. U.S.A. 94: 861-866.

53. Enholm B, Paavonen K, Ristimaki A, et al. (1997) Comparison of VEGF, VEGF-B, VEGF-C and Ang-I mRNA regulation by serum, growth factors, oncoproteins and hypoxia. Oncogene 14: 2475-2483.

54. White FC, Benehacene A, Scheele JS, et al. (1997) VEGF mRNA is stabilized by ras and tyrosine kinase oncogenes, as well as by UV radiation-evidence for divergent stabilization pathways. Growth Factors 14: 199-212.

55. Shirasawa S, Furuse M, Yokoyama N, et al. (1993) Altered growth of human colon cancer cell lines disrupted at activated Ki-ras. Science 260: 85-88.

56. Viloria-Petit AM, Rak J, Hung M-C, et al. (1997) Neutralizing antibodies against EGF and ErbB-2/ neu receptor tyrosine kinases down-regulate VEGF production by tumor cells in vitro and in vivo: angiogenic implications for signal transduction therapy of solid tumors. Am. J. Pathol. 151: 1523-1530.

57. Goldman CK, Kim J, Wong WL, et al. (1993) Epidermal growth factor stimulates vascular endothelial growth factor production by human malignant glioma cells: A model of glioblastoma multiforme pathophysiology. Mol. Biol. Cell 4: 121-133.

58. Goad DL, Rubin J, Wang H, et al. (1996) Enhanced expression of vascular endothelial growth factor in human SaOS-2 osteoblast-like cells and murine osteoblasts induced by insulin-like growth factor I. Endocrinology 137: 2262-2268.

59. Kevil CG, De Benedetti A, Payne DK, et al. (1996) Translational regulation of vascular permeability factor by eukaryotic initiation factor 4E: Implications for tumor angiogenesis. Int. J. Cancer 65: 785-790.

60. Zabrenetzky V, Harris CC, Steeg PS, et al. (1994) Expression of the extracellular matrix molecule thrombospondin inversely correlates with malig- 
nant progression in melanoma, lung and breast carcinoma cell lines. Int. J. Cancer 59: 191-195.

61. Sheibani N, Frazier WA. (1996) Repression of thrombospondin-l expression, a natural inhibitor of angiogenesis, in polyoma middle $\mathrm{T}$ transformed NIH3T3 cells. Cancer Lett. 107: 45-52.

62. Siemeister G, Weindel K, Mohrs K, et al. (1996) Reversion of deregulated expression of vascular endothelial growth factor in human renal carcinoma cells by von Hippel-Lindau tumor suppressor protein. Cancer Res. 56: 2299-2301.

63. Mukhopadhyay D, Knebelmann B, Cohen HT, et al. (1997) The von Hippel-Lindau tumor suppressor gene product interacts with $\mathrm{Spl}$ to repress vascular endothelial growth factor promoter activity. Mol. Cell. Biol. 17: 5629-5639.

64. Shweiki D, Itin A, Soffer D, et al. (1992) Vascular endothelial growth factor induced by hypoxia may mediate hypoxia-initated angiogenesis. $\mathrm{Na}$ ture 359: 843-845.

65. Shweiki D, Neeman M, Itin A, et al. (1995) Induction of vascular endothelial growth factor expression by hypoxia and by glucose deficiency in multicell spheroids: Implications for tumor angiogenesis. Proc. Natl. Acad. Sci. U.S.A. 92: 768-772.

66. Ikeda E, Achen MG, Breier G, et al. (1995) Hy- poxia-induced transcriptional activation and increased mRNA stability of vascular endothelial growth factor in C6 glioma cells. J. Biol. Chem. 270: 19761-19766.

67. Stein I, Neeman M, Shweiki D, et al. (1995) Stabilization of vascular endothelial growth factor mRNA by hypoxia and hypoglycemia and coregulation with other ischemia-induced genes. Mol. Cell. Biol. 15: 5363-5368.

68. D'Amore PA, Shima DT. (1996) Tumor angiogenesis: a physiological process or genetically determined? Cancer Metastasis Rev. 15: 205-212.

69. Thompson TC, Southgate J, Kitchener G, et al. (1989) Multistage carcinogenesis induced by ras and $m y c$ oncogenes in a reconstituted organ. Cell 56: 917-930.

70. Fan Z, Mendelsohn J. (1998) Therapeutic application of anti-growth factor receptor antibodies. Curr. Opin. Oncol. 10: 67-73.

71. Bais C, Santomasso B, Coso O, Arvanitakis L, Raaka EG, Gutkind JS, Asch AS, Cesarman E, Gerhengorn MC, Mesri EA. (1998) G-proteincoupled receptor of Kaposi's sarcoma-associated herpesvirus is a viral oncogene and angiogenesis activator. Nature 391: 86-89. 